\title{
Changes in Porosity, Electrical and Surface Properties after Laundering of Heat-treated AgNW/PDMS/PU Nanofiber-web
}

\author{
Eugene Lee, Sujin Cha, and Gilsoo Cho* \\ Department of Clothing and Textiles, Yonsei University, Seoul 03722, Korea \\ (Received September 1, 2020; Revised November 1, 2020; Accepted November 3, 2020)
}

\begin{abstract}
In this study, to investigate the effects of heat treatment and polydimethylsiloxane (PDMS) coating on durability to laundering, the changes in the porosity, electrical resistance, and surface property of the heat-treated silver nanowire $(\mathrm{AgNW}) / \mathrm{PDMS} /$ polyurethane $(\mathrm{PU})$ nanofiber-web before and after laundering were examined. To prepare the conductive specimens, PU nanofiber-web, $1 \mathrm{wt} \%$ of AgNW dispersion in ethanol, and two types of PDMS precursors were used. A total of four specimens were prepared: Specimen A (The AgNW/PU nanofiber-web), Specimen AH (The heat-treated AgNW/PU nanofiber-web), Specimen AP (The PDMS-coated AgNW/PU nanofiber-web), and Specimen AHP (The heat-treated AgNW/PDMS/PU nanofiber-web). The specimen was rinsed and dehydrated after laundering under the conditions according to ISO 6330. To investigate the changes of porosity after a single washing cycle, mean pore diameter and pore size distribution were measured. Linear electrical resistance and microscopic surface view of the specimen were evaluated. As a result, many micro-pores were distributed in Specimen AH because of the heat treatment. After laundering, Specimen AH still had the micro-pores, and thus, the heat treatment improved the durability to laundering. Also, the electrical resistance of Specimen AH was only changed slightly even after laundering, which was because the heat treatment strengthened the bonding between the nanofibers and the silver nanowires, thus the silver nanowires sufficiently remained on the specimen surface even after laundering. And, Specimen AH showed that the silver nanowires formed a network even after laundering and were evenly covered with silver nanowires onto the specimen surface. This result affected the aforementioned electrical performance, and in fact, the electrical resistance of Specimen AH was the lowest regardless of laundering. However, it was impossible to measure the porosity of Specimen AP and Specimen AHP due to the PDMS coating. Therefore, the heat treatment affected the durability to laundering as well as the electrical conductivity to increase, but PDMS coating blocked the micro-pores of the nanofiber-web and enabled higher initial resistance.
\end{abstract}

Keywords: Polyurethane nanofiber-web, Silver nanowire, Heat treatment, Durability to laundering, Electronic-textile

\section{Introduction}

In the post-COVID-19 era, as the 4th industrial revolution evolved in combination with IT technology, hyper-connected systems such as e-commerce, home-healthcare, and hometraining have emerged. Especially, the demand for smart clothing that can monitor vital signals such as heart rate for daily life is rapidly increasing. Smart clothing is a new platform that can detect and analyze the wearer's bio-signals while having its own property as a textile material [1].

Textile sensor is drawing attention in the smart clothing field [2], which refers to a sensor that detects physiological signals using electronic textiles (e-textiles). Textile sensors for smart clothing have several requirements: they must be flexible and wearable, and have sufficient electrical conductivity and low contact impedance. At the same time, basic properties of textile materials such as adequate strength and elongation, and durability to laundering should be maintained. However, the current textile sensors or etextiles have a problem that causes the conductive particles to be peeled off during laundering. The problem not only leads to a decrease in electrical conductivity and performance deterioration of the devices, but also is harmful to humans $[3,4]$. Therefore, in this study, we tried to improve the

*Corresponding author: gscho@yonsei.ac.kr electrical conductivity and durability to laundering of the etextiles by using heat treatment and coating process.

Nanofibers usually have $100 \mathrm{~nm}$ or less of diameter, but textile nanofibers are generally thinner than $1,000 \mathrm{~nm}$ or submicrons because they easily lose textile required properties and have a limited use of extremely thin nanofibers. Nanofibers are available as a form of nanofiber-web mainly produced via electrospinning, centrifugal spinning, and melt-blown spinning techniques $[5,6]$. Nanofiber-web is a kind of nonwovens and consisted of randomly positioned nanofibers. Due to its high surface area and tremendous number of micro-pores, the treatment is very efficiency during solution process and it makes the electrical and mechanical properties improved for smart textiles [6-8]. Polyurethane (PU) nanofiber-web is an ultra-light and ultrathin membrane, and also has flexibility and breathability compared to the other textiles.

Our team has recently conducted the researches [8-12] that electrical conductivity was imparted to PU nanofiber-web by using silver nanowires (AgNWs). Kim et al. [9] reported that the AgNW/PU nanofiber-web had $22 \Omega / \mathrm{cm}$ of electrical resistance, $0.65 \mathrm{MPa}$ of tensile strength, and $0.00486 \mathrm{~g} \cdot \mathrm{cm}$ of stiffness. Lee and Cho [8] revealed that heat treatment affected a decrease in the electrical resistance and an improvement of the tensile strength and durability. Lee et al. [10] reported that the nanofiber-web was coated with 
polydimethylsiloxane (PDMS), thereby the tensile strength and elongation were improved. However, in the previous studies [8-12], durability to laundering, which is one of the basic textile properties, was not evaluated. Although some previous studies [13-17] dealt with the durability to laundering or washability of conductive nanofibers, the specimens were simply immersed in distilled water. Besides, the effects of washing time and temperature, and stirring speed on electrical and physical properties of conductive nanofibers were examined, but the effect of repeated washing cycles on their washability was not discussed. In this study also to confirm washability of the conductive nanofiber-web, one cycle of laundering process was conducted. In particular, this study had the distinction of evaluating the durability to laundering under the standard conditions reflecting the actual laundering environment unlike other related previous studies conducted. Thus, in this study, we tried to compare changes in porosity, electrical resistance, and surface property of the heat-treated AgNW/PDMS/PU nanofiberweb before and after a laundering cycle.

\section{Experimental}

\section{Materials}

A commercially available PU nanofiber-web was kindly provided by Pardam, s.r.o. Ltd., Czech Republic. The basic specifications of the nanofiber-web are presented in Table 1. The fiber diameters ranged from $500 \mathrm{~nm}$ to $1 \mu \mathrm{m}$, with a weight of $13 \mathrm{~g} \mathrm{~m}^{-2} .1 \mathrm{wt} \%$ of AgNW dispersed in ethanol (purity: $>95 \%$ ) was purchased from KLK Co., Republic of

Table 1. Characteristics of polyurethane nanofiber-web

\begin{tabular}{ccccc}
\hline Name & $\begin{array}{c}\text { Fiber diameter } \\
(\mathrm{nm})\end{array}$ & $\begin{array}{c}\text { Thickness } \\
(\mu \mathrm{m})\end{array}$ & $\begin{array}{c}\text { Weight } \\
\left(\mathrm{g} / \mathrm{m}^{2}\right)\end{array}$ & $\begin{array}{c}\text { Manufacturing } \\
\text { technique }\end{array}$ \\
\hline $\begin{array}{c}\text { PU } \\
\text { Nanofiber-web }\end{array}$ & $500-1,000$ & 20 & 13 & $\begin{array}{c}\text { Centrifugal } \\
\text { spinning }\end{array}$ \\
\hline
\end{tabular}

Table 2. Treatment conditions of the conductive specimens

\begin{tabular}{cccc}
\hline \multirow{2}{*}{ Specimen } & \multicolumn{3}{c}{ Treatment condition } \\
\cline { 2 - 4 } & AgNW & Heat treatment & PDMS coating \\
\hline UT & $\mathrm{X}$ & $\mathrm{X}$ & $\mathrm{X}$ \\
A & $\mathrm{O}$ & $\mathrm{X}$ & $\mathrm{X}$ \\
$\mathrm{AH}$ & $\mathrm{O}$ & $50^{\circ} \mathrm{C}, 24 \mathrm{~h}$ & $\mathrm{X}$ \\
$\mathrm{AP}$ & $\mathrm{O}$ & $\mathrm{X}$ & $80^{\circ} \mathrm{C}, 2 \mathrm{~h}$ \\
AHP & $\mathrm{O}$ & $50^{\circ} \mathrm{C}, 24 \mathrm{~h}$ & $80^{\circ} \mathrm{C}, 2 \mathrm{~h}$ \\
\hline
\end{tabular}

Korea. The length and diameter of the silver nanowires were $20-25 \mu \mathrm{m}$ and 30-35 $\mathrm{nm}$, respectively. The two types of PDMS precursors, pre-polymer part A, and the curing agent part B (SYLGARD 184, Dow Corning) were used for the coating process.

\section{Preparation of Specimens}

Four different specimens were prepared and named as A, AH, AP and AHP. First, Specimen A was prepared by dipcoating PU nanofiber-web with AgNWs to impart the electrical conductivity. A $20 \times 20 \mathrm{~cm}^{2}$ of nanofiber-web was dipped in an AgNWs bath, and the treated specimen was dried at room temperature for 24 hours [9]. Then, Specimen $\mathrm{AH}$ was obtained by treating specimen A with heat. For specimen $\mathrm{AH}$, a nanofiber-web dipped in an AgNWs bath was heat-treated at $50{ }^{\circ} \mathrm{C}$ for 24 hours in a vacuum oven (OV-11, Jeio Tech Co., Ltd., Republic of Korea) [8]. For specimen AP, a PU nanofiber-web was treated with AgNWs and dried at room temperature for 24 hours, and then PDMS (10:1 of part A and part B ratio) were applied by a doctor blade (SH 1117/200, Sheen Instruments, Republic of Korea), and cured at $50{ }^{\circ} \mathrm{C}$ for 2 hours [18]. And, for specimen AHP, a PU nanofiber-web treated with AgNWs and dried at $50{ }^{\circ} \mathrm{C}$ for 24 hours in a vacuum oven was applied with PDMS using a doctor blade and cured at the same conditions. These three specimens (Specimen AH, AP, and AHP) were prepared to improve the durability of the specimen's electrical conductivity. The treatment conditions of the four specimens were summarized in Table 2.

\section{Laundering Tests}

According to the testing conditions of ISO 6330 (Domestic washing and drying procedures for textile testing) [19,20], the specimen was rinsed and dehydrated after laundering under the conditions shown in Table 3. After a single washing cycle, the specimen was naturally dried at room temperature (Flat dry). After the laundering test, the weight of the specimen was evaluated by an analytical balance (Explorer Analytical Balance, Ohaus, USA) and compared between before and after the washing. Also, the percentage change was calculated using the following equation to compare the change of the porosity, electrical and surface properties of the specimens before and after the laundering test. V1 is the initial value, that is, before the laundering test. $\mathrm{V} 2$ is the final value, that is, after the laundering test.

$$
\% \text { change }(\%)=(\mathrm{V} 2-\mathrm{V} 1) /|\mathrm{V} 1| \times 100
$$

Table 3. Conditions for laundering test

\begin{tabular}{|c|c|c|c|c|c|c|c|c|}
\hline \multirow[b]{2}{*}{ Procedure } & \multicolumn{4}{|c|}{ Washing } & \multicolumn{2}{|c|}{ Rinse } & \multicolumn{2}{|c|}{ Spin } \\
\hline & $\begin{array}{c}\text { Total load } \\
\text { (air-dry mass) (kg) }\end{array}$ & $\begin{array}{l}\text { Temp. } \\
\left({ }^{\circ} \mathrm{C}\right)\end{array}$ & $\begin{array}{l}\text { Liquor level } \\
(\mathrm{mm})\end{array}$ & $\begin{array}{l}\text { Wash time } \\
(\mathrm{min})\end{array}$ & $\begin{array}{l}\text { Liquor level } \\
(\mathrm{mm})\end{array}$ & $\begin{array}{l}\text { Rinse time } \\
\quad(\min )\end{array}$ & $\begin{array}{l}\text { Spin speed } \\
(\mathrm{rpm})\end{array}$ & $\begin{array}{l}\text { Spin time } \\
\text { (min) }\end{array}$ \\
\hline & $2 \pm 0.1$ & $27 \pm 3$ & $297 \pm 25$ & 8 & $297 \pm 25$ & 3 & $399-420$ & 4 \\
\hline
\end{tabular}




\section{Evaluation of Mean Pore Diameter and Pore Size Distri- bution}

To investigate the changes of porosity after the laundering test, mean pore diameter and pore size distribution were measured by Capillary flow porometer (1100-AEHXL, Porous Media Inc., USA). The size of the specimen was $2 \times 2 \mathrm{~cm}^{2}$, and Galwick (surface tension=15.4 dynes $/ \mathrm{cm}$, Porous Material Inc., USA) was used as a wetting fluid.

\section{Measurement of Electrical Resistance}

To evaluate the electrical property, linear electrical resistance of the specimen was measured using a multimeter (Donghwa DM1010, Republic of Korea). The positions of the two probes of the multimeter were placed randomly on the specimen surface. The distance between two probes was set to $1 \mathrm{~cm}$ and measurements were repeated 5 times. To investigate the relationship between the mean pore diameter of the specimen and the electrical resistance, regression analysis was conducted using SPSS (ver. 24.0) program.

\section{Analysis of Surface Property}

To examine the surface property, microscopic view of the specimen surface was analyzed by a field emission scanning electron microscopy (FE-SEM, JSM-6701F, JEOL Ltd., USA).

\section{Results and Discussion}

\section{Comparison of Porosity, Electrical and Surface Properties of the Specimens}

In this study, the porosity, electrical and surface properties of the four specimens were discussed, and then, the changes in these properties of the specimens before and after laundering were compared. As a result of the porosity (Figure 1), the untreated PU nanofiber-web (Specimen UT) had 10.66 microns of mean pore diameter. The mean pore diameter of Specimen A and Specimen AH was 4.34 microns and 3.78 microns, respectively. Compared to Specimen UT, the mean pore diameter of the treated specimens was lower. This was because the pores size between the polyurethane nanofibers decreased as the silver nanowires were treated on the specimen surface. In particular, many micro-pores were distributed in Specimen AH compared to Specimen A, which can be inferred that more silver nanowires were attached to the surface of Specimen AH by heat treatment. However, in the case of Specimen AP and Specimen AHP, it was impossible to measure the porosity of the specimens. This was because the pores between nanofibers were completely covered by the PDMS coating. Thus, although the PDMS coating treatment increased the strength and durability of the conductive nanofiber-web [10], it had a limitation causing the micropores of the specimens to be blocked.

As a result of the electrical property (Figure 2), Specimen

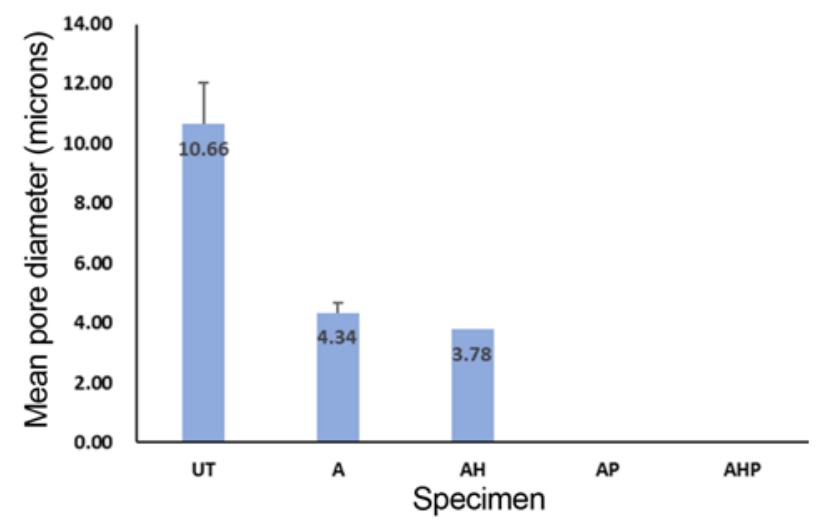

Figure 1. Mean pore diameter of the PU nanofiber-web and the conductive specimens.

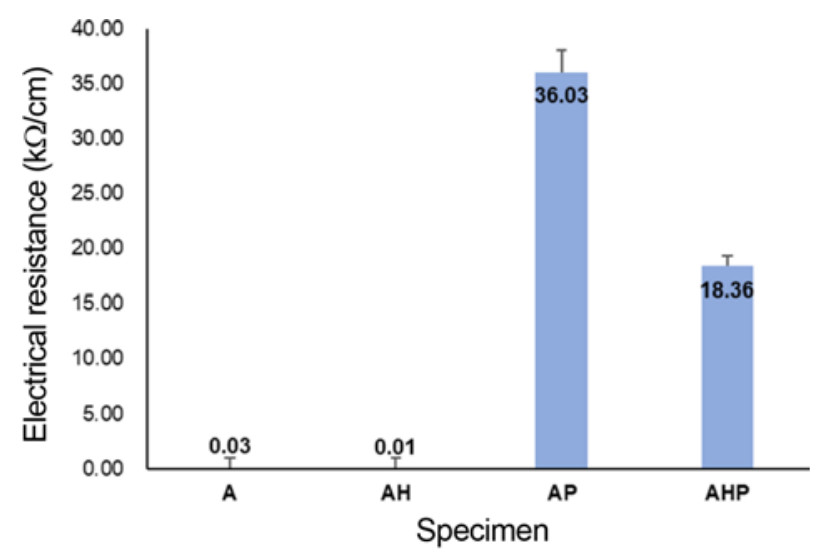

Figure 2. Electrical resistance of the conductive specimens.

A had $0.03 \mathrm{k} \Omega / \mathrm{cm}$ of electrical resistance, and the resistance of Specimen AH was $0.01 \mathrm{k} \Omega / \mathrm{cm}$. Specimen AH showed the lowest resistance, which was because a silver nanowire network was formed on the specimen surface and electrons could easily flow since the ethanol was completely evaporated by heat treatment [9]. This not only affected the electrical resistance, but also the aforementioned pore size, and thus Specimen AH had the smallest mean pore diameter and the best electrical performance. Specimen AP and Specimen AHP had $36.03 \mathrm{k} \Omega / \mathrm{cm}$ and $18.36 \mathrm{k} \Omega / \mathrm{cm}$ of resistance, respectively. Compared to Specimen A and Specimen AH, the resistance of both specimens dramatically increased because of PDMS coating treatment, which was consistent with the result of the previous study [18].

And, in the image of the surface of Specimen UT (Figure 3 ), it was observed that the polyurethane nanofibers were randomly arranged. However, the nanofibers were not observed in all four treated specimens. In Specimen A and Specimen $\mathrm{AH}$, the silver nanowires were evenly coated on the surface of the specimen. In particular, in the case of Specimen AH, the silver nanowires were coated densely on the specimen surface while forming a network between the 

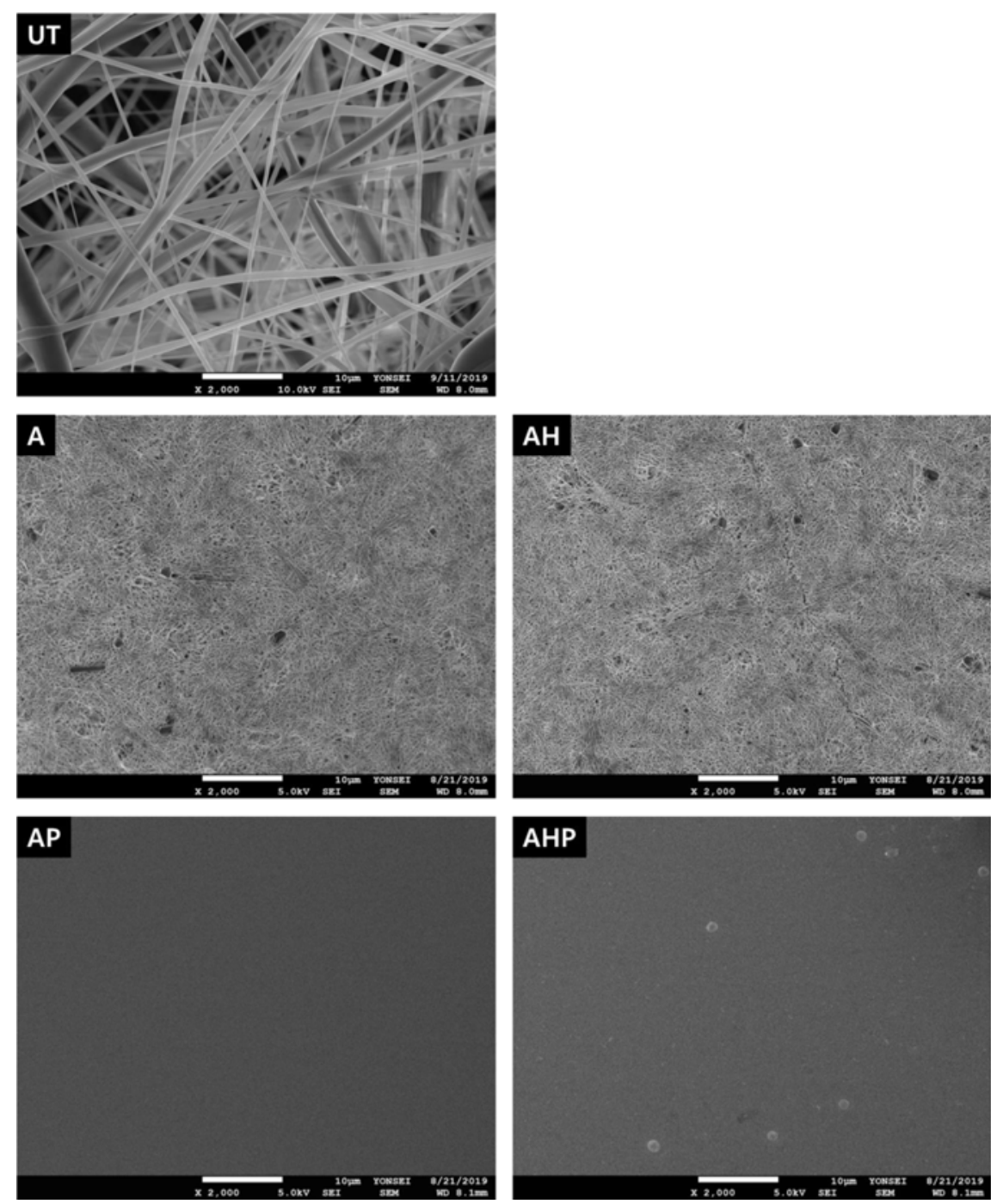

Figure 3. FE-SEM images of the PU nanofiber-web and the conductive specimens.

silver wires due to heat treatment. This result clarified the reason that Specimen $\mathrm{AH}$ had a smaller mean pore diameter than that of Specimen A discussed above. In Specimen AP and Specimen AHP, not only the polyurethane nanofibers but also the silver nanowires were not observed at all, but had smooth and flattened surfaces since both surfaces of the specimens were coated thickly with PDMS.

\section{Changes of Mean Pore Diameter and Pore Size Distribu- tion after Laundering}

To investigate how the porosity of the specimen changed after one washing cycle, the mean pore diameter and the pore size distribution were measured. As a result, after the laundering of Specimen A and Specimen $\mathrm{AH}$, the mean pore diameter was 5.09 microns and 4.28 microns (Table 4), respectively, and the pore diameter increased by $17 \%$ and $13 \%$ (Figure 4), respectively compared to the specimens
Table 4. Mean pore diameter of the specimens before and after laundering

\begin{tabular}{cccccc}
\hline & \multicolumn{2}{c}{ Before laundering } & & \multicolumn{2}{c}{ After laundering } \\
\cline { 2 - 3 } \cline { 5 - 6 } Specimen & $\begin{array}{c}\text { Mean pore } \\
\text { diameter } \\
\text { (microns) }\end{array}$ & $\begin{array}{c}\text { Standard } \\
\text { deviation }\end{array}$ & & $\begin{array}{l}\text { Mean pore } \\
\text { diameter } \\
\text { (microns) }\end{array}$ & $\begin{array}{c}\text { Standard } \\
\text { deviation }\end{array}$ \\
\hline A & 4.34 & 1.39 & & 5.09 & 0.42 \\
AH & 3.78 & 0.34 & & 4.28 & 0.55 \\
AP & - & - & & - & - \\
AHP & - & - & & - \\
\hline
\end{tabular}

before laundering. It can be inferred that some silver nanowires densely covered on the specimen surface were detached and peeled off during laundering, thereby the pore size of the specimens increased after laundering. However, 


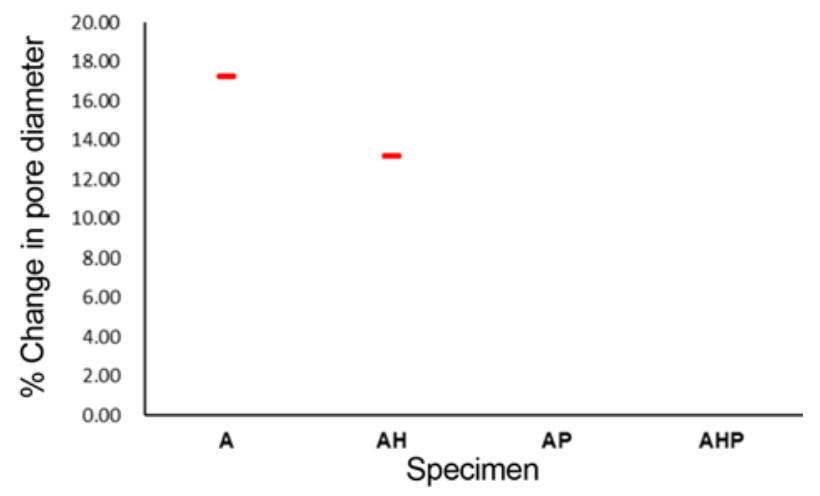

Figure 4. Changes in the mean pore diameter of the conductive specimens after laundering.

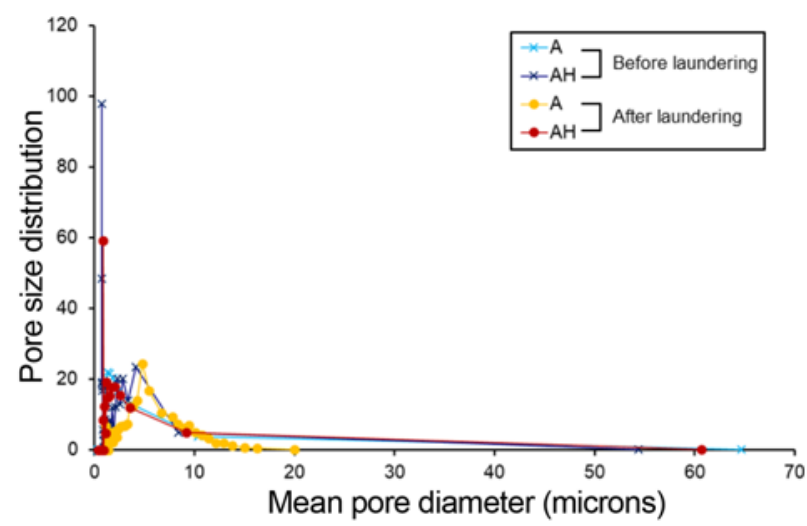

Figure 5. Comparison of the pore size distribution of the conductive specimens before and after laundering.

as shown in Figure 5, the micro-pores were still distributed in Specimen $\mathrm{AH}$ even after laundering, thus, the heat treatment improved the durability to laundering $[15,17]$. In the case of Specimen AP and Specimen AHP, it was impossible to measure their porosity regardless of laundering since the pores between the nanofibers were completely covered by the PDMS coating.

\section{Changes of Electrical Resistance after Laundering}

The electrical resistance of the specimens before and after laundering was compared. As a result, Specimen AH had $1.92 \mathrm{k} \Omega / \mathrm{cm}$ of resistance even after laundering, which indicated that Specimen AH still had the lowest resistance among the four specimens (Table 5). As a result of the percentage change in the resistance after laundering (Figure 6), Specimen AH showed that the resistance only changed slightly even after laundering compared to Specimen A. This was because the heat treatment caused the bonding between the nanofibers and the silver nanowires to be strengthened, thus the silver nanowires sufficiently remains on the specimen surface even after laundering (Table 6). In the case of Specimen AP and Specimen AHP, the percentage change
Table 5. Electrical resistance of the specimens before and after laundering

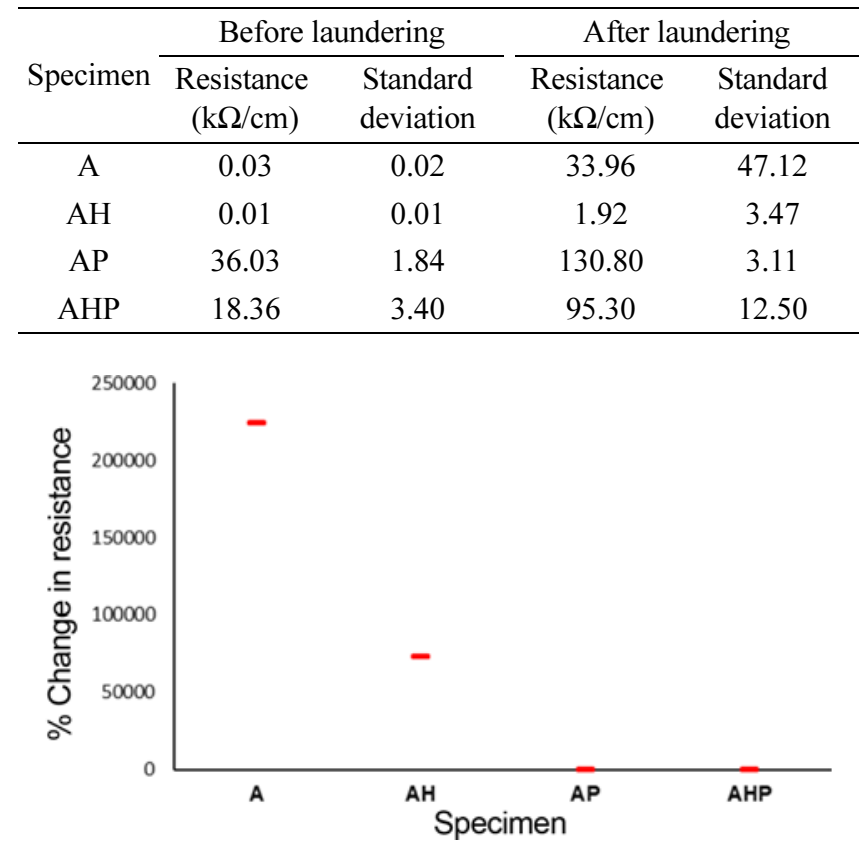

Figure 6. Changes in the electrical resistance of the conductive specimens after laundering.

Table 6. Weight of the specimens before and after laundering

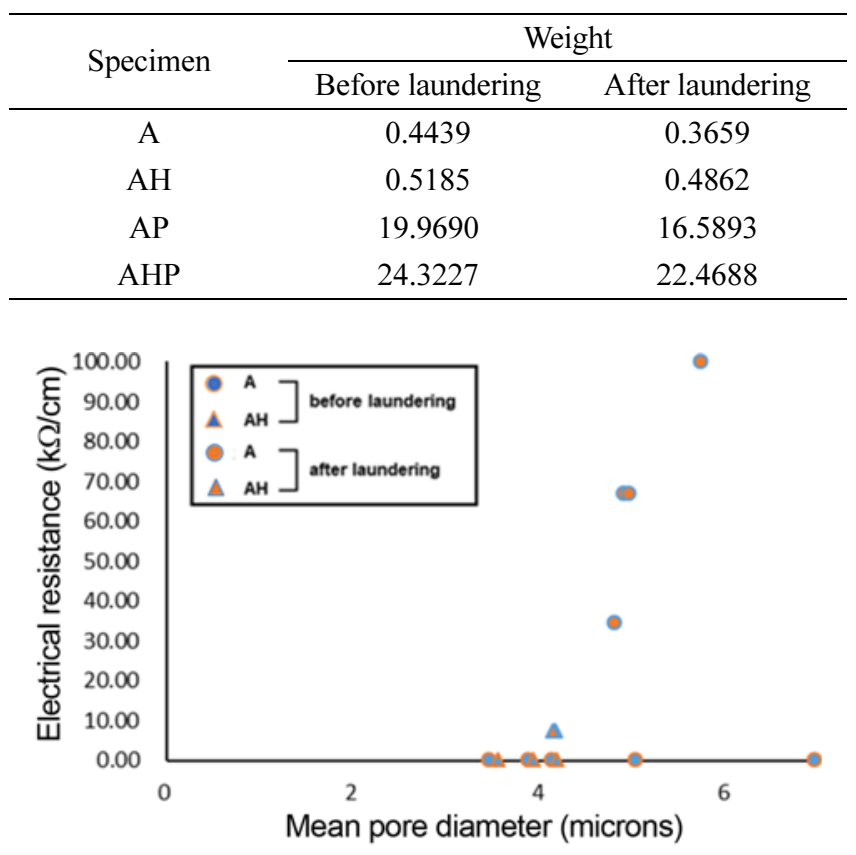

Figure 7. Relationship between mean pore diameter and electrical resistance of the conductive specimens before and after laundering.

in the resistance after laundering drastically decreased compared to the other specimens (Figure 6) due to the PDMS coating treatment. However, PDMS treatment caused 
the initial resistance to be increased and all pores of the nanofiber-web to be completely covered as aforementioned. Also, in this study, the relationship between the mean pore diameter of the specimen and the electrical resistance was analyzed as presented in Figure 7. As a result, as the mean pore diameters of the specimen increased, the electrical resistance also tends to increase $\left(\mathrm{R}^{2}=.83\right)$. This can be inferred that as the number of silver nanowires that formed a kind of membrane onto the specimen surface were more, it affected the pore sizes to be smaller, which eventually improved the electrical conductivity.

\section{Surface Property Changes after Laundering}

The changes in the surface property of the specimen
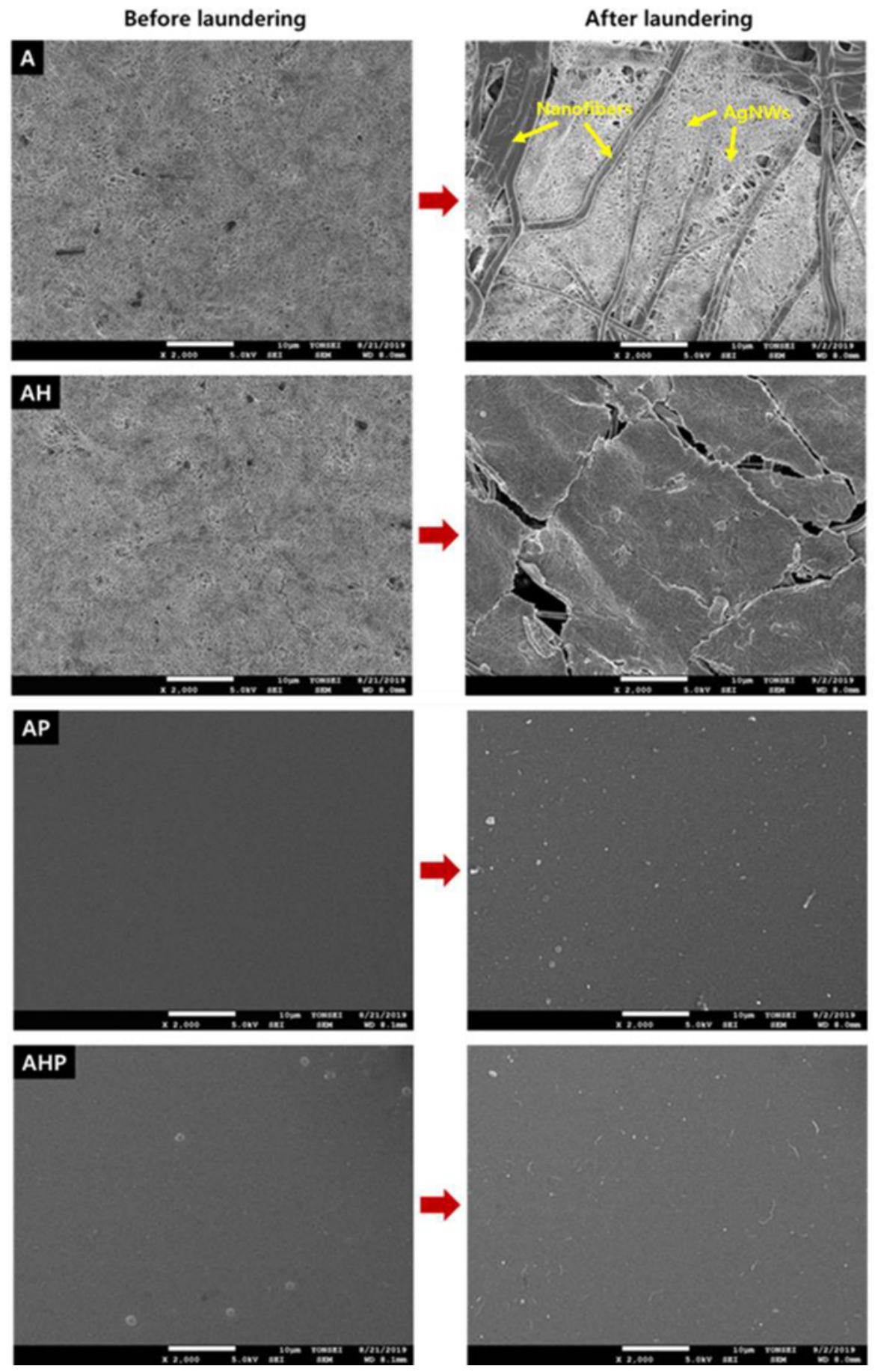

Figure 8. Comparison of the surface property of the conductive specimens before and after laundering. 
before and after laundering were examined. As a result, Specimen A was observed to have the silver nanowires detached and peeled off by laundering (Figure 8), thereby the polyurethane nanofibers were exposed. On the other hand, in the case of Specimen AH, it showed that the silver nanowires formed a network even after laundering and were evenly covered with silver nanowires onto the specimen surface. In particular, the surface properties at low and high magnification (Figure 9) showed that Specimen AH was evenly covered with silver nanowires onto the entire surface even after laundering. This result affected the aforementioned electrical resistance, and in fact, according to the result of the resistance, the electrical resistance of Specimen AH was the lowest regardless of laundering. In the case of Specimen
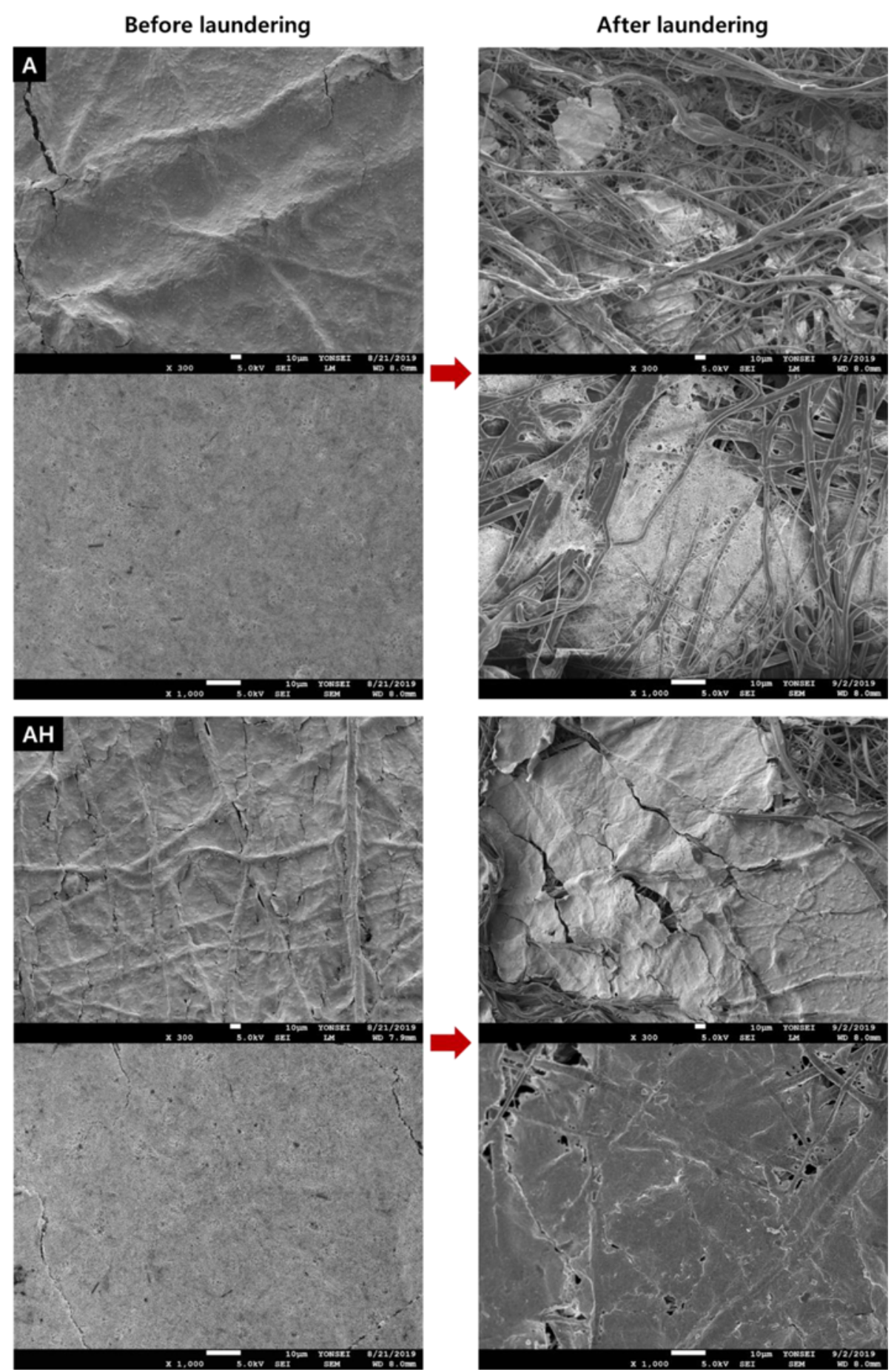

Figure 9. SEM images of Specimen A and Specimen AH at two different magnifications: $\times 300$ and $\times 1,000$. 
AP and Specimen AHP, the surface appearance of the specimen was not changed significantly after laundering because of PDMS coating treatment. As discussed before, the PDMS coating improved durability to laundering, however it caused blocked micro-pores and high initial resistance increase.

\section{Conclusion}

In this study, how the pore size distribution, mean pore diameter, electrical resistance, and surface property of the specimens changed after one washing cycle was investigated. The results showed that Specimen AH still had many micropores even after laundering. Also, Specimen AH had the lowest resistance and maintained low resistance well even after laundering. This was because the heat treatment strengthened the bonding between the polyurethane nanofibers and the silver nanowires, and thus, the silver nanowires sufficiently remained on the surface of the specimen after a laundering cycle. In other words, the heat treatment improved the durability to laundering of the specimens. Therefore, the heat-treated AgNW/PU nanofiber-web showed well maintained electrical performance, while a silver nanowire network was formed on the specimen surface and the mean pore diameter was micro-sized even after one washing cycle. However, in further study, a repeated laundering test should be conducted to discuss the durability to laundering in depth. Prior to this, it is necessary to establish a standard test method and procedure of durability to laundering for nanofiber-web based smart textiles, and to evaluate the durability to laundering of conductive nanofiberweb compliance with the standard. Also, researches on the resistance against abrasion and friction are necessary to evaluate the conductive nanofiber-web in terms of the maintenance aspect of daily life smart clothing.

\section{Acknowledgment}

This research was supported by Basic Science Research Program through the National Research Foundation of Korea (NRF) funded by the Ministry of Education, Science and Technology (No. NRF-2016R1A2B4014668) and the Brain Korea 21 Plus Project of Dept. of Clothing and Textiles, Yonsei University in 2020. This work was supported (in part) by the Yonsei University Research Fund (Post Doc. Researcher Supporting Program) of 2020 (project no.: 2019-12-0133).

\section{References}

1. G. Cho, K. Jeong, M. J. Paik, Y. Kwun, and M. Sung, IEEE Sens. J., 11, 3183 (2011).

2. E. Lee and G. Cho, Smart Mater. Struct., 28, 045004 (2019).

3. J. O. Park, C. Park, S. Choi, and O. Y. Park, "Proceedings of the Korean Society of Disaster Information", p.123, 2017.

4. N. M. Van der Velden, K. Kuusk, and A. R. Köhler, Mater. Des., 84, 313 (2015).

5. L. Persano, A. Camposeo, C. Tekmen, and D. Pisignano, Macromol. Mater. Eng., 298, 504 (2013).

6. S. Ramakrishna, "An Introduction to Electrospinning and Nanofibers", pp.7-21, World Scienctific, Singapore, 2005.

7. J. H. Kim and J. S. Lee, Fiber. Polym., 19, 2394 (2018).

8. E. Lee and G. Cho, Text. Res. J., 89, 2938 (2019).

9. I. Kim, E. Lee, E. Jang, and G. Cho, Text. Res. J., 88, 1215 (2018)

10. H. C. Lee, E. Lee, and G. Cho, Smart Mater. Struct., 29, $075028(2020)$.

11. E. Lee, I. Kim, H. Liu, and G. Cho, Fiber. Polym., 18, 1749 (2017).

12. W. Kim, E. Lee, J. Choi, and G. Cho, Fiber. Polym., 21, $1195(2020)$.

13. J. Y. Won, M. H. Jee, S. H. Park, Y. G. Jeong, and D. H. Baik, Text. Sci. Eng., 46, 1 (2009).

14. X. Wu, Y. Han, X. Zhang, and C. Lu, ACS Appl. Mater. Inter, , 8, 9936 (2016).

15. V. Babaahmadi, M. Montazer, and W. Gao, Carbon, 118, 443 (2017).

16. C. Zhang, S. Fan, H. Shao, X. Hu, B. Zhu, and Y. Zhang, Carbon, 148, 16 (2019).

17. H. Li, W. Zhang, Q. Ding, X. Jin, Q. Ke, Z. Li, D. Wang, and C. Huang, ACS Appl. Mater. Inter., 11, 38023 (2019).

18. I. Kim and G. Cho, Smart Mater. Struct., 27, 075006 (2018).

19. Y. Yang and G. Cho, "Novel Stretchable Textile-Based Transmission Bands: Electrical Performance and Appearance After Abrasion/Laundering, and Wearability", in Proceedings of the 13th International Conference on Human-Computer Interaction International 2009, pp.806813, San Diego, 2009.

20. ISO 6330:2012(en), Textiles — Domestic Washing and Drying Procedures for Textile Testing, 2012. 